\title{
A dichotomy between HSB and LSB galaxies
}

\author{
Marc Verheijen ${ }^{1}$ \\ National Radio Astronomy Observatory, Socorro, New Mexico \\ Brent Tully \\ University of Hawaii, Institute for Astronomy, Honolulu, Hawaii
}

\begin{abstract}
.
A complete sample of spiral galaxies in the Ursa Major cluster is imaged at various optical wavelengths and in the Near-Infrared $K^{\prime}$-band. HI rotation curves were obtained for all gas rich systems. The Near-Infrared surface brightness distribution of disk galaxies turns out to be bimodal; galaxies avoid a domain around $\mu_{0}^{\mathrm{i}}\left(\mathrm{K}^{\prime}\right) \approx 18.5 \mathrm{mag} / \operatorname{arcsec}^{2}$. This bimodality is particularly striking when only the more isolated, non-interacting systems are considered. The Luminosity Function of the HSB family of galaxies is truncated well above the completion limit while the Luminosity Function of the LSB family is still sharply rising at our limiting magnitude. Near-Infrared mass-to-light ratios suggest that HSB galaxies are close to a kinematic maximum-disk situation while LSB galaxies are dark matter dominated at all radii. Assuming equal Near-Infrared massto-light ratios for both HSB and LSB systems, we find that the gap in the surface brightness distribution corresponds to a situation in which the baryonic mass is marginally self-gravitating. We finally conclude that the luminosity-line width relation is a fundamental correlation between the amount and distribution of dark matter mass and the total luminosity, regardless of how the luminous mass is distributed within the dark mater halo.
\end{abstract}

\section{Introduction}

The distribution of disk central surface brightnesses is determined with some confidence in the B-band. It shows a steep rise at the bright end and remains roughly flat towards fainter surface brightnesses (McGaugh et al, 1995; McGaugh, 1996; de Jong, 1996; Bothun et al, 1997 and references therein). In general, however, this distribution is determined using B-band photometry and the observed surface brightnesses are not or obscurely corrected for the effects of inclination and internal extinction. Furthermore, the effects of metallicity and age of a stellar population on the B-band surface brightness of a galaxy

${ }^{1}$ Jansky Fellow, Array Operations Center 
can be considerable. It might not be surprising that the observed B-band surface brightness distribution is flat to some extent since large galaxy-to-galaxy variations in dust content, age and metallicity will smooth out any underlying, physically interesting distribution like the stellar surface density or angular momentum distribution. Therefore, "...it is possible that a great deal of our present understanding about the surface brightness distribution of galaxies is based on a parameter that is very insensitive to the actual physical characteristics of galaxies" (Davies, 1990).

In our talks we presented results from a Near-Infrared imaging survey of a complete volume limited sample of galaxies in the Ursa Major Cluster. In the Near-Infrared, internal extinction is neglegible and the age and metallicity effects on the luminosity of a stellar population are minimal. We show that the distribution of Near-Infrared face-on disk central surface brightnesses is bimodal; there is a relative lack of spirals with $\mu_{0}^{\mathrm{i}}\left(\mathrm{K}^{\prime}\right) \approx 18.5^{\mathrm{m}}$. Analyzing HI rotation curves shows that this gap in the Near-Infrared surface brightness distribution may correspond to a dynamical instability when a disk is marginally self-gravitating.

\section{The Sample and Observations}

The Ursa Major cluster, located in the Supergalactic Plane, consists of 80 galaxies in a $\sim 80 \mathrm{Mpc}^{3}$ volume at a distance of $15.5 \mathrm{Mpc}$. This volume is overdense by roughly a factor 10 compared to the average field. However, the population of galaxies is comparable to the field. It comprises mainly spiral galaxies, a dozen S0 and maybe one elliptical system. A complete sample of 62 galaxies, intrinsically brighter then the SMC, has been identified in the B-band. Since all galaxies are at the same distance, there is little question about their relative luminosities and disk scale lengths. This cluster is described in detail by Tully et al (1996).

Surface photometry in the B, R and I passbands was obtained for all galaxies in 11 observing runs between February 1984 and March 1996. Photometric K'band images of 69 spiral galaxies in Ursa Major were obtained in May 1991, February 1992 and March 1993 using a $256^{2} \mathrm{HgCdTe}$ detector on the 24-inch and 88-inch telescopes of the University of Hawaii on Mauna Kea. Sixty galaxies form a nearly complete sample (only U6628 and U7129 have missing K'-band data) of spirals intrinsically brighter than the SMC. Images and luminosity profiles can be found in Tully et al (1996).

HI synthesis data for all galaxies with sufficient HI gas were obtained with the Westerbork Synthesis Radio Telescope between 1991 and 1996. From these data, HI rotation curves were derived by fitting tilted rings to the HI velocity fields. The rotation curves were corrected for the effects of beam smearing by overlaying them on position-velocity diagrams and adjusting them by eye. The radial surface density profiles of the gas were derived from the total HI maps by averaging the flux in ellipses following the adjusted tilted rings fits. The HI data are described in detail by Verheijen (1997). 

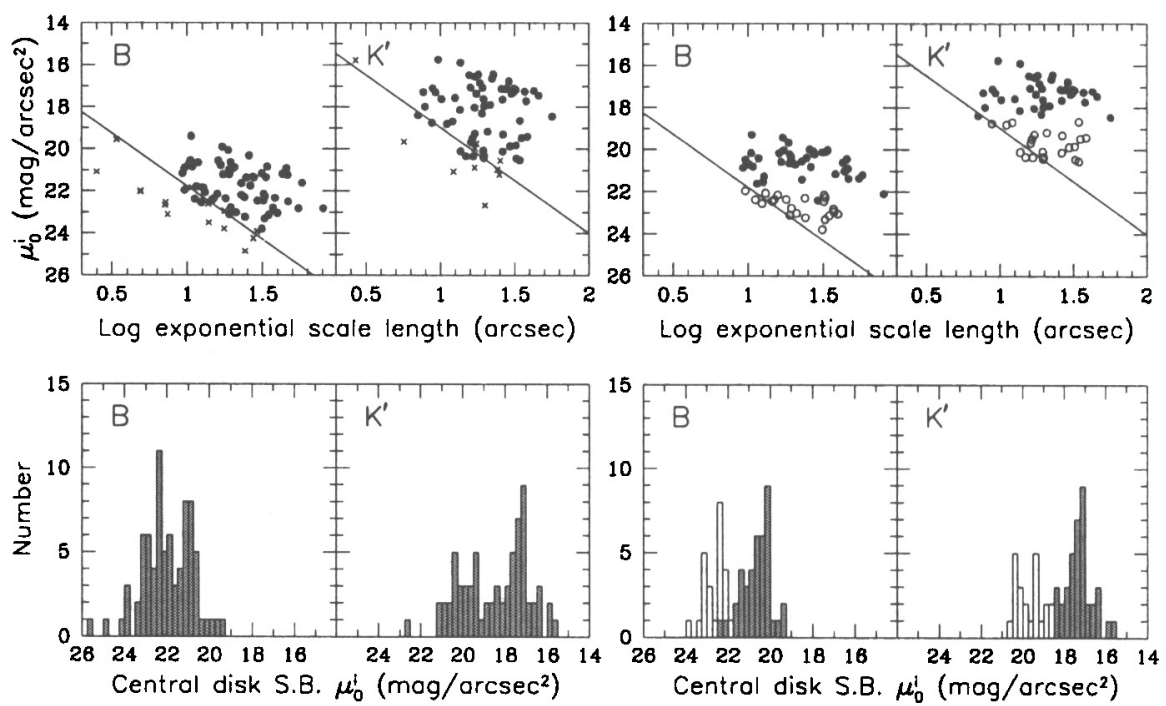

Figure 1. Upper-left panels: Plots of central disk surface brightness versus the $\log$ of the exponential disk scale length as measured in the $B$ and $K^{\prime}$ bands. Galaxies are assumed to be transparent in both passbands $\left(C^{\mathrm{B}, \mathrm{K}^{\prime}}=1\right)$. Slanted lines indicate the completion limit for purely exponential disks. Crosses indicate galaxies fainter than the SMC. Upper-right panels: Complete sample only while the LSB family of galaxies is identified with open symbols. Here, $C_{\mathrm{HSB}, \mathrm{LSB}}^{\mathrm{K}^{\prime}}=1$ while $C_{\mathrm{HSB}}^{\mathrm{B}}=0.23$ to correct for internal extinction and $C_{\mathrm{LSB}}^{\mathrm{B}}=1$. Lower panels: Histogram distributions of the central disk surface brightnesses corresponding to the upper panels.

\section{Surface Brightness Distributions}

From the calibrated optical and Near-Infrared images, luminosity profiles were extracted by averaging the flux density in concentric ellipses of equal ellipticity and position angle. Exponential disk fits were made by fitting straight lines to the quasi-linear part of the luminosity profiles, yielding disk scale lengths and central surface brightnesses. A possible upturn due to a bulge in the inner parts of the profiles was carefully excluded from the fit. The following discusssion of the photometric results will be restricted to the $\mathrm{B}$ - and $\mathrm{K}^{\prime}$-band data.

\subsection{A Bimodal Distribution}

The measured central surface brightnesses $\mu_{0}(\lambda)$ at a certain wavelength $\lambda$ were corrected for inclination and internal extinction according to

$$
\mu_{0}^{\mathrm{i}}(\lambda)=\mu_{0}(\lambda)-2.5 C^{\lambda} \log (\mathrm{b} / \mathrm{a})
$$

where $C^{\lambda}$ accounts for internal extinction. If galaxies are transparent, $C^{\lambda}=1$ and $\mu_{0}(\lambda)$ will only be corrected for the path length through the inclined disk 


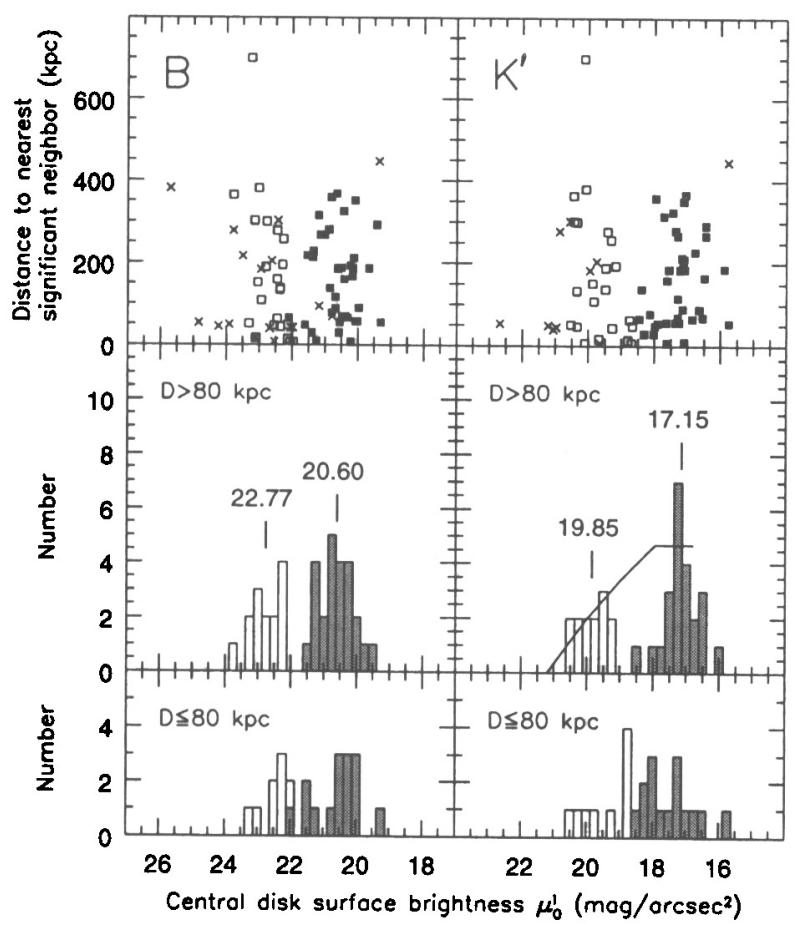

Figure 2. Upper panels: Disk central surface brightness in the $\mathrm{B}$ and $\mathrm{K}^{\prime}$ passbands versus projected distance to nearest significant neighbor. Nearly all galaxies with intermediate surface brightness have a close companion. Middle panels: Surface brightness distributions for galaxies more isolated than $80 \mathrm{kpc}$ from their nearest neighbor. The line in the $\mathrm{K}^{\prime}$-band panel roughly indicates the completion limit based on purely exponential disks. Lower panels: Surface brightness distributions for galaxies with close companions (in projection).

while $C^{\lambda}<1$ takes a correction for internal extinction into account. Values of $\mu_{0}^{\mathrm{i}}$ are plotted against disk scale lengths in Figure 1. LSB galaxies are indicated with open symbols in the right panels in which a trend of surface brightness with total luminosity can be seen.

We assume that galaxies are transparent in the Near-Infrared and thus use $C^{\mathrm{K}^{\prime}}=1$. Doing so, we find an apparent lack of galaxies with $\mu_{0}^{\mathrm{i}}\left(\mathrm{K}^{\prime}\right) \approx 18.5$ $\mathrm{mag} / \operatorname{arcsec}^{2}$ (see $\mathrm{K}^{\prime}$-panels in Figure 1). This is a first hint to a possible bimodality in the Near-Infrared surface brightness distribution. We use the NearInfrared data to define a low surface brightness galaxy in case its $\mu_{0}^{\mathrm{i}}\left(\mathrm{K}^{\prime}\right)>18.5$ $\mathrm{mag} / \operatorname{arcsec}^{2}$ and a high surface brightness galaxy if $\mu_{0}^{\mathrm{i}}\left(\mathrm{K}^{\prime}\right)<18.5 \mathrm{mag} / \mathrm{arcsec}^{2}$.

It should be pointed out that de Jong (1996) obtained K'-band images of 85 galaxies in his sample and derived a Near-Infrared surface brightness distribution without a hint of any bimodality. Unfortunately, his sample is dominated 
by HSB galaxies and the volume corrections for the LSB systems are quite uncertain.

The internal extinction in the B-band gives rise to much more uncertainty in any correction of $\mu_{0}(\mathrm{~B})$ to face-on. Figure 1 shows the distribution of $\mu_{0}^{\mathrm{i}}(\mathrm{B})$ assuming that either $C^{\mathrm{B}}=1$ for all galaxies (left $\mathrm{B}$-band panels) or $C^{\mathrm{B}}=1$ only for LSB galaxies while $C^{\mathrm{B}}=0.23$ for HSB galaxies (right $\mathrm{B}$-band panels). The latter assumption is based on the notion that the overall fainter LSB galaxies in Ursa Major are in general not detected by IRAS, indicating a low dust content. Given this bimodal extinction correction in the B-band, the resulting bimodality of the B-band surface brightness distribution is largely artificial. It should be noted that the former assumption, $C^{\mathrm{B}}=1$ for all galaxies, leads to a flat surface brightness distribution in the B-band.

\subsection{A Near-Neighbor Effect}

Investigating whether the LSB galaxies in our sample are more isolated than the HSB galaxies lead to an interesting result. In the upper panels of Figure 2 we plot the surface brightness of a galaxy versus the projected distance to its nearest significant neighbor; the nearest galaxy with an I-band luminosity of at least $10 \%$ of the galaxy considered. It turns out that galaxies of intermediate surface brightness, $\mu_{0}^{\mathrm{i}}\left(\mathrm{K}^{\prime}\right) \approx 18.5 \mathrm{mag} / \operatorname{arcsec}^{2}$, have near companions and are often involved in a tidal interaction. Considering the more isolated galaxies, the bimodality in the surface brightness distribution is striking (middle panels). The line in the middle $\mathrm{K}^{\prime}$-panel indicates how our completion limit maps onto the surface brightness distribution. Within our sample, the LSB galaxies are not more isolated than the HSB galaxies although the most isolated system in our sample is an LSB.

\section{The Luminosity Functions.}

This bimodality of HSB and LSB families is also reflected in the Luminosity Functions. Figure 3 shows a linear version of the Luminosity Functions in both the $\mathrm{B}$ and $\mathrm{K}^{\prime}$ bands including galaxies fainter than the completion limit. The filled part of the histogram corresponds to the HSB family of galaxies. The Luminosity Functions of the HSB galaxies clearly cut off well above the completion limit while the Luminosity Functions of the LSB galaxies keep rising toward the faint end until the completion limit is reached. In the $\mathrm{K}^{\prime}$-band, the HSB and LSB Luminosity Functions are more separated than in the B-band. This may result in a shallow dip in the Luminosity Functions around $M_{K^{\prime}}^{b, i}=-21.5^{m}$ while the B-band Luminosity Function is quite flat.

\section{Stellar Mass-to-Light Ratios}

We derived extended rotation curves of $22 \mathrm{HI}$-rich, non-interacting and sufficiently inclined galaxies in the complete sample which allow us to investigate a possible relation between this Near-Infrared surface brightness bimodality and the mass-to-light ratios of the stellar populations and kinematics of the stellar disks. The HI rotation curves were decomposed using the $\mathrm{K}^{\prime}$ luminosity pro- 

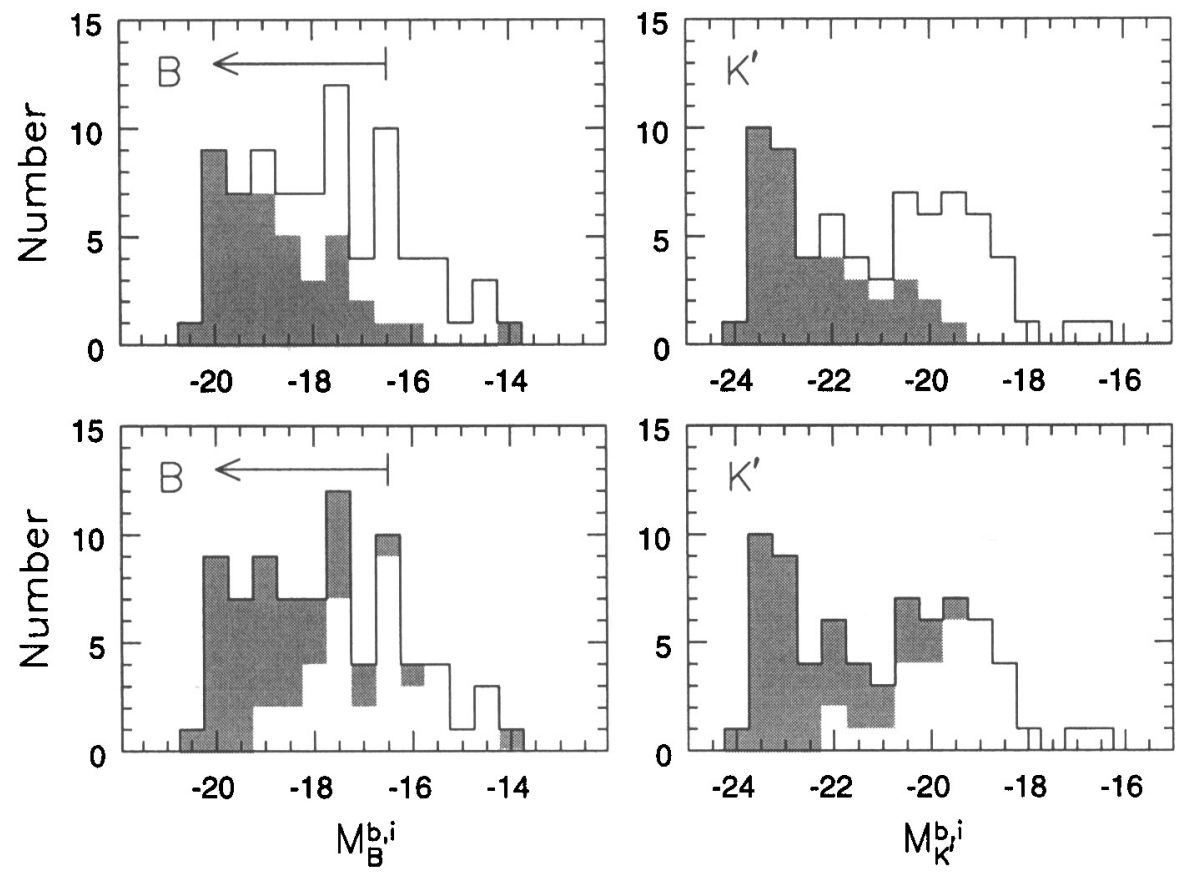

Figure 3. Luminosity Functions in the $\mathrm{B}$ and $\mathrm{K}^{\prime}$ passbands including all identified cluster members. The open part of the histograms correspond to the LSB family. The horizontal arrow in the B-band panels indicates the complete sample. The Luminosity Function of the HSB galaxies drops well above the completion limit. The sub-histograms are switched between the upper and lower panels.

files, the HI surface density profiles and an isothermal sphere model for the dark matter halo (Figure 5). Maximum-disk fits were made, avoiding a hollow halo core. The resulting stellar mass-to-light ratios in the $\mathrm{K}^{\prime}$-band are plotted versus $\mathrm{B}-\mathrm{K}^{\prime}$ color in the upper panels of Figure 4.

First, let's consider the results for the HSB galaxies (filled symbols). In the $\mathrm{K}^{\prime}$-band, the scatter in the stellar mass-to-light ratios of the HSB galaxies is small $(0.22)$ and the mean value of $\left\langle\mathrm{M}_{*} / \mathrm{L}_{\mathrm{K}^{\prime}}\right\rangle_{\mathrm{HSB}}=0.71$ (excluding NGC 3992) is in accordance with stellar population models (dashed lines) by Bruzual and Charlot (1993). Furthermore, for the HSB galaxies, there is a clear trend of the B-band mass-to-light ratios with $\mathrm{B}-\mathrm{K}^{\prime}$ color as would be expected from stellar population models. From a stellar population point-of-view the maximum-disk hypothesis seems to make sense for HSB galaxies. Note that if HSB galaxies are systematically half-maximum-disk, the small $\mathrm{K}^{\prime}$-band scatter would remain but the average value would drop to $<\mathrm{M}_{*} / \mathrm{L}_{\mathrm{K}^{\prime}}>_{\mathrm{HSB}}=0.18$ and the trend of $\left(\mathrm{M}_{*} / \mathrm{L}_{\mathrm{B}}\right)$ with $\mathrm{B}-\mathrm{K}^{\prime}$ color would become too shallow.

The LSB galaxies on the other hand show a much larger scatter, have a significantly higher average mass-to-light ratio in the $\mathrm{K}^{\prime}$-band and do not 

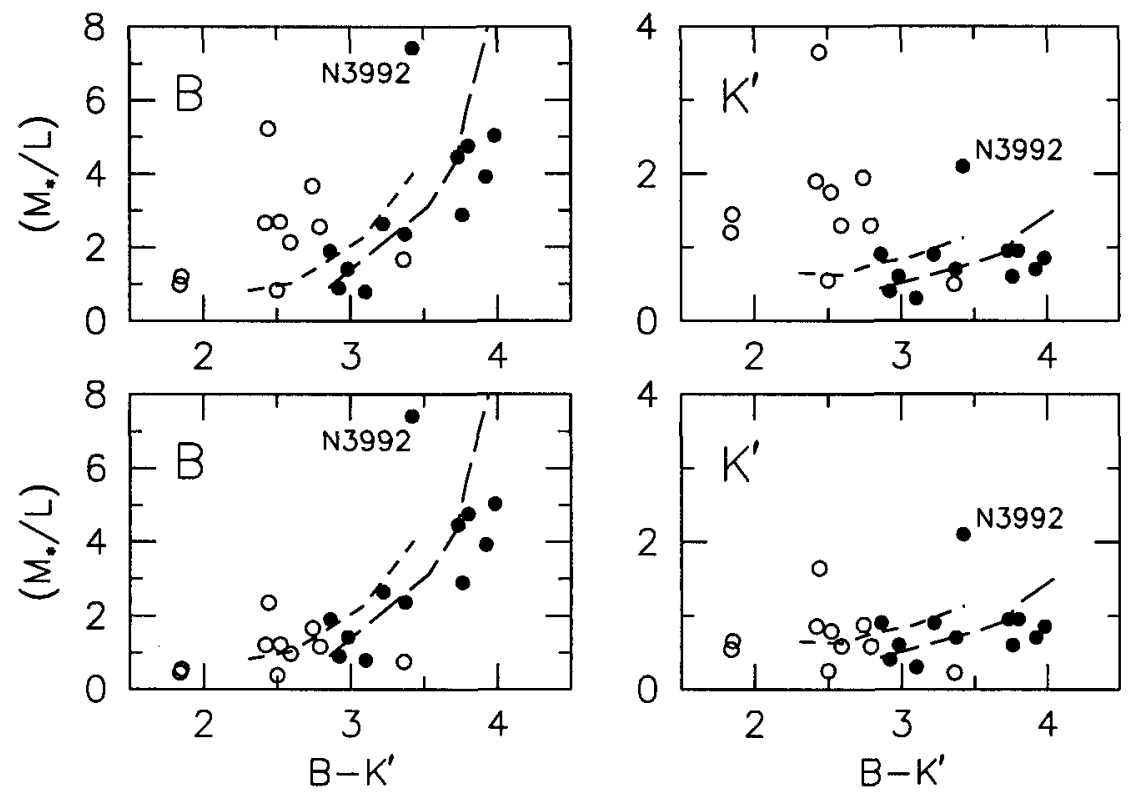

Figure 4. Stellar mass-to-light ratios in the $B$ - and $K^{\prime}$-band as a function of B-K' color for HSB (filled symbols) and LSB galaxies (open symbols). Upper panels: Mass-to-light ratios as derived from maximum-disk fits for both HSB and LSB galaxies. Lower panels: Same as upper panels for the HSB galaxies but the $K^{\prime}$-band mass-to-light ratios of the LSB galaxies were scaled down such that $<\mathrm{M}_{*} / \mathrm{L}_{\mathrm{K}^{\prime}}>_{\mathrm{LSB}}=\left\langle\mathrm{M}_{*} / \mathrm{L}_{\mathrm{K}^{\prime}}\right\rangle_{\mathrm{HSB}}=0.71$. The dashed lines indicate expectations from the Bruzual and Charlot (1993) stellar population models with solar metallicities, a Salpeter IMF and ages of 2-17 Gyr. Long dashes: single burst, short dashes: exponential SFR.

show any trend of $\left(\mathrm{M}_{*} / \mathrm{L}_{\mathrm{B}}\right)$ with $\mathrm{B}-\mathrm{K}^{\prime}$ color. From this we conclude that we overestimate the mass-to-light ratios for LSB galaxies and, consequently, that it is unlikely that LSB galaxies in general are in a maximum-disk situation. This is also supported by the fact that the higher mass-to-light ratios for LSB galaxies can be understood easily from a geometrical point-of-view as explained by Zwaan et al (1995).

The lower panels of Figure 4 show the mass-to-light ratios in case the HSB galaxies have a maximum-disk (same as upper panels) but the $\mathrm{K}^{\prime}$-band mass-to-light ratios of the LSB galaxies are scaled down such that the average mass-to-light ratios of $\mathrm{HSB}$ and LSB galaxies are the same; $\left\langle\mathrm{M}_{*} / \mathrm{L}_{\mathrm{K}^{\prime}}\right\rangle_{\mathrm{LSB}}=$ $<\mathrm{M}_{*} / \mathrm{L}_{\mathrm{K}^{\prime}}>_{\mathrm{HSB}}=0.71$. Although the scatter in $\left(\mathrm{M}_{*} / \mathrm{L}_{\mathrm{K}^{\prime}}\right)$ of the LSBs is still somewhat larger than that of the HSB galaxies, the trend of $\left(\mathrm{M}_{*} / \mathrm{L}_{\mathrm{B}}\right)$ with $\mathrm{B}-\mathrm{K}^{\prime}$ color seems to be continued by the LSB galaxies in the lower left panel. The luminosities of both HSB and LSB galaxies were corrected for internal extinction in similar ways. 

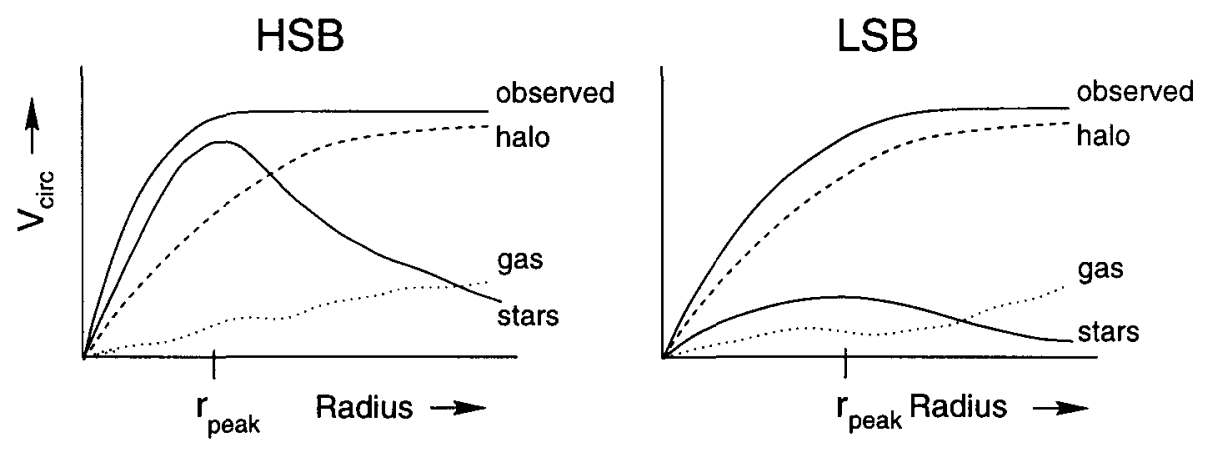

Figure 5. Schematic difference between the kinematics of an HSB and an LSB galaxy with a similar observed maximum rotational velocity.

\section{A Dynamical Instability}

Next, we investigate the relative dynamical importance of the dark matter halo, assuming equal stellar mass-to-light ratios in the $\mathrm{K}^{\prime}$-band for both HSB and LSB galaxies. Again, we decomposed the HI rotation curves but this time we assumed $\left(\mathrm{M}_{*} / \mathrm{L}_{\mathrm{K}^{\prime}}\right)=0.6$ for all galaxies. This mass-to-light ratio is slightly less than the average in order not to violate the maximum-disk situation too badly for most systems. We calculated for each stellar disk the maximum rotational velocity $V_{\text {disk }}^{\max }$ which occurs at $r_{\text {peak }}$ around 2.1 disk scale lengths and the rotational velocity $V_{\text {halo }}\left(r_{\text {peak }}\right)$ induced by the dark matter halo, at the same radius where the rotation curve of the stellar disk peaks. If $V_{\text {disk }}^{\max }>V_{\text {halo }}\left(r_{\text {peak }}\right)$ then the stellar disk dominates the potential in the inner regions while the dark matter dominates if $V_{\text {disk }}^{\max }<V_{\text {halo }}\left(r_{\text {peak }}\right)$. The contributions by the gas component to the potential have been ignored. Figure 6 shows the measured ratios $V_{\text {disk }}^{\max } / V_{\text {halo }}\left(r_{\text {peak }}\right)$.

Again, we find a bimodal distribution along the lines of the HSB/LSB dichotomy; HSB disks are self-gravitating and close to a maximum-disk situation while the potentials of LSB galaxies are dominated by the dark matter halo at all radii. Furthermore, it seems that stellar disks avoid a situation in which the disk is marginally self-gravitating $V_{\text {disk }}^{\max } \approx V_{\text {halo }}\left(r_{\text {peak }}\right)$. This seems to hint at an instability, either in the dynamics of an established dissipationless stellar disk or during the formation process of a galaxy when it consisted mostly of dissipational gas. Testing the hypothesis of a dynamical instability in a marginally self-gravitating baryonic disk would require $\mathrm{N}$-body (hydro-)dynamical simulations at a very high dynamic range.

\section{Summary}

Our findings and conclusions can be summarized as follows. 


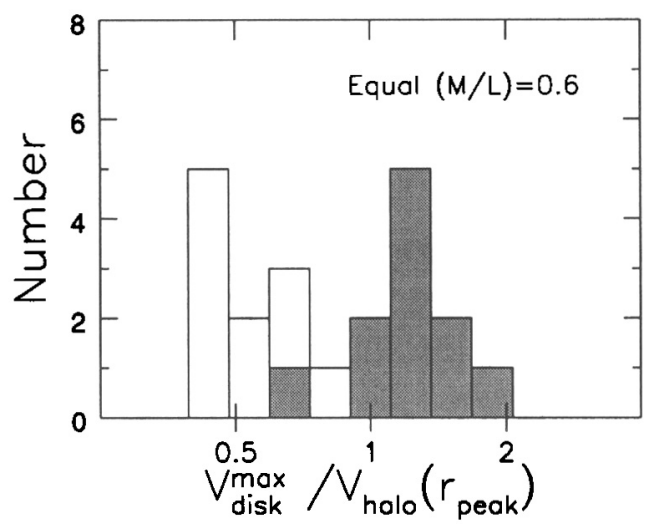

Figure $6 . \quad$ Ratio of the maximum rotational velocity induced by the stellar disk $V_{\text {disk }}^{\max }$ over the rotational velocity induced by the halo at $r_{\text {peak }}$ where $V_{\text {disk }}^{\text {max }}$ occurs. The filled histogram is for HSB galaxies, the open histogram for LSB galaxies. An equal stellar mass-to-light ratio in the $K^{\prime}$-band of 0.6 is assumed for all galaxies. NGC 3992 is omitted.

- There is an avoidance of a domain $\left(\mu_{0}^{\mathrm{i}}\left(\mathrm{K}^{\prime}\right) \approx 18.5 \mathrm{mag} / \operatorname{arcsec}^{2}\right)$ of NearInfrared disk central surface brightness. For isolated galaxies, there is a distinct gap (factor 10 in density) between HSB and LSB families.

- The Luminosity Function of the HSB family is essentially truncated faintward of $M_{B}=-17^{\mathrm{m}}$. The LSB family Luminosity Function is sharply increasing at our completion limit of $M_{B}=-16.5^{\mathrm{m}}$. There might be a shallow dip in the $K^{\prime}$-band Luminosity Function around $M_{K^{\prime}}=-21.5^{m}$.

- Although HSB and LSB galaxies with similar $\left(\mathrm{L}, \mathrm{V}_{\max }\right)$ lie at the same position on the luminosity-line width relation, they may lie in very different domains in surface brightness-scale lengths plots.

- Maximum-disk decompositions of HSB rotation curves give reasonable Near-Infrared stellar mass-to-light ratios (average of 0.7 with $\mathrm{rms}$ scatter of only 0.2 ) and an obvious trend of B-band mass-to-light ratio with $\mathrm{B}-\mathrm{K}^{\prime}$ color. Maximum-disk decompositions of LSB galaxies give mass-tolight ratios with a higher average, a larger scatter and no trend with color. We infer that HSB disks are close to maximum-disk and self-gravitating inside $\sim 2$ scale lengths while LSB galaxies are well below maximum-disk and dark matter dominated at all radii.

- Assuming that both HSB and LSB galaxies have similar Near-Infrared mass-to-light ratios, we infer that galaxies avoid a situation in which their baryonic matter is marginally self-gravitating.

- The $\mathrm{V}^{\max }$ that is relevant for the luminosity-line width relation is given by the mass distribution of the dark matter halo while the total luminosity of the dissipational matter is in fixed proportion to the halo mass. The exact 
distribution of the luminous matter within the dark matter halo is irrelevant for the luminosity-line width relation. In high angular momentum LSB systems, the dissipational matter reaches its rotational equilibrium at large radii and the mass distribution of dark matter remains dominant. In low angular momentum HSB systems, the dissipational matter collapses enough to become self-gravitating and might even give rise to a rotation curve which is declining in the inner parts before it becomes flat in the dark matter supported outer regions.

\section{Future work}

One might argue that our statistics are not overwhelming or that our findings are particular to the Ursa Major cluster. To tackle both issues we are currently analyzing the results of a blind VLA survey in the Perseus-Pisces ridge which yields an HI selected and volume limited sample of galaxies of both high and low surface brightness. The volume surveyed is similar to the entire Ursa Major volume. Near-Infrared surface photometry is planned for the fall of 1999 which should give us the Near-Infrared surface brightness distribution of this HI selected sample. In this way we hope to avoid any optical selection effects that might be strongly biased in favor of HSB systems. The combined data sets will yield a sample that is twice as large as the current Ursa Major sample itself.

Of course, we eagerly await the results of any other ongoing project aimed at measuring the Near-Infrared surface brightness distribution.

Acknowledgments. Mike Pierce, Jia-Sheng Huang and Richard Wainscoat participated in the collection of the data. The Westerbork Synthesis Radio Telescope is operated by the Netherlands Foundation for Research in Astronomy, with financial support by the Netherlands Organization for Scientific Research (NWO). This research has been supported by NATO Collaborative Research Grant 940271 and grants from the US National Science Foundation.

\section{References}

Bothun, G.D., Impey, C.D. and McGaugh, S.S., 1997, PASP, 109, 745

Bruzual, G.A. and Charlot, S., 1993, ApJ, 405, 538

Davies, J.I, 1990, MNRAS, 244, 8

de Jong, R.S., 1996, A\&A, 313, 45

McGaugh, S.S., Bothun, G.D. and Schombert, J.M., 1995, AJ, 110, 573

McGaugh, S.S., 1996, MNRAS, 280, 337

Tully, R.B., Verheijen, M.A.W., Pierce, M.J., Huang, J.-S. and Wainscoat, R.J., 1996, AJ, 112,2471

Tully, R.B. and Verheijen, M.A.W., 1997, ApJ, 484, 145

Verheijen, M.A.W., 1997, PhD thesis, University of Groningen

Zwaan, M.A., van der Hulst, J.M., de Blok, W.J.G. and McGaugh, S.S., 1995, MNRAS, 237, L35 